THÉORIE DES NOMBRES

BESANÇON

Une remarque sur la capitulation du groupe des classes au sens restreint

C. MAIRE 


\title{
UNE REMARQUE SUR LA CAPITULATION DU GROUPE DES CLASSES AU SENS RESTREINT
}

\author{
CHRISTIAN MAIRE
}

\begin{abstract}
On prosose de montrer que le groupe des classes au sens restreint de certains corps quadratiques réels $k$ capitule entièrement dans une sous-extension stricte du corps de Hilbert au sens restreint de $k$.
\end{abstract}

\section{RÉSULTAT PRINCIPAL}

Soient $k$ un corps de nombres et $c l_{k}^{+}$le groupe des classes de $k$ au sens restreint. Par le théorème de l'idéal principal, on sait que $\mathrm{cl}_{k}^{+}$capitule entièrement dans $k_{1}^{+}$, le corps de Hilbert au sens restreint de $k$.

On montre ici le résultat suivant :

Théorème 1.1. Soit $k$ un corps quadratique réel, dont la norme de l'unité fondamentale est 1. Si la 2-partie de $c_{k}^{+}$est $(2,2)$, alors $c_{k}^{+}$capitule dans une sous-extension stricte de $k_{1}^{+}$.

Remarque 1.2. Il suffit de montrer que la 2-partie $\mathrm{cl}_{k}^{+}(2) \mathrm{de}_{\mathrm{cl}}^{+}{ }_{k}^{+}$capitule dans une extension quadratique de $k$.

\section{QUELQUES RAPPELS}

2.1. Le groupe $c l_{k}^{+}$. Le groupe des classes au sens restreint est par définition

$$
c l_{k}^{+}=\frac{I_{k}}{P_{k}^{+}}
$$

où $I_{k}$ est le groupe des idéaux fractionnaires non nuls de $k$, et où $P_{k}^{+}$est le sous-groupe de $I_{k}$ des idéaux principaux totalement positifs, c'est à dire si $(\alpha) \in P_{k}^{+}, \alpha \in k^{\times}$, alors tous les plongements réels de $\alpha$ sont positifs.

On sait alors que le groupe $c l_{k}^{+}$est isomorphe au groupe de Galois $\operatorname{Gal}\left(k_{1}^{+} / k\right)$, ceci par l'application d'Artin, où $k_{1}^{+}$est l'extension abélienne maximale de $k$ non ramifiée aux places finies.

Si $c l_{k}$ désigne le groupe des classes de $k$, on a la formule suivante :

$$
\left|c l_{k}^{+}\right|=\left|c l_{k}\right| \frac{2^{r_{1}}}{\left[E_{k}: E_{k}^{+}\right]},
$$

où $r_{1}$ est le nombre de places réelles de $k, E_{k}$ est le groupe des unités de $k$, et $E_{k}^{+}$le sous-groupe des unités de $k$, totalement positives.

Date: 11 juin 1998. 
2.2. Un peu de théorie des genres. Soit $k$ un corps quadratique réel, tel que $c l_{k}^{+}(2)=(2,2)$.

Nous allons montrer que $k_{1}^{+}(2) / \mathbb{Q}$ est abélienne.

Tout d'abord, il est clair que $k_{1}^{+}(2) / \mathbb{Q}$ est galoisienne, ceci par maximalité de $k_{1}^{+}(2) / k$. Notons alors $M$ le corps des genres relatif à $k_{1}^{+}(2) / \mathbb{Q}$, c'est à dire, $M / \mathbb{Q}$ est l'extension abélienne maximale de $\mathbb{Q}$ contenue dans $k_{1}^{+}(2)$. En particulier, $M$ contient $k$.

On a la suite exacte (cf. [6]) :

$$
1 \rightarrow \frac{E_{\mathbb{Q}}^{+}}{E_{\mathbb{Q}}^{+} \cap \mathcal{N}_{k / \mathbb{Q}}} \longrightarrow \bigoplus_{v} I_{v}(k / \mathbb{Q}) \longrightarrow \operatorname{Gal}(M / \mathbb{Q}) \longrightarrow 1
$$

où $E_{\mathbb{Q}}^{+} \cap \mathcal{N}_{k / \mathbb{Q}}$ sont les unités de $\mathbb{Q}$, positives, normes locales partout dans $k / \mathbb{Q}$, et où $I_{v}(k / \mathbb{Q})$ est le groupe d'inertie dans $k / \mathbb{Q}$ pour une place $v$ de $\mathbb{Q}$.

Notons que $\operatorname{Gal}(k / \mathbb{Q})=\langle\tau\rangle$ agit trivialement $\operatorname{sur} \operatorname{Gal}(M / k)(\operatorname{car} M / \mathbb{Q}$ est abélienne) ; par maximalité de $M, \operatorname{Gal}(M / k)$ est le plus grand quotient de $c l_{k}^{+}(2)$ sur lequel $G a l(k / \mathbb{Q})$ agit trivialement. Ainsi

$$
G a l(M / k)=\frac{c l_{k}^{+}(2)}{c l_{k}^{+}(2)^{1-\tau}}=\frac{c l_{k}^{+}(2)}{c l_{k}^{+}(2)^{2}}=c l_{k}^{+}(2) .
$$

Il vient alors :

- $M=k_{1}^{+}(2)$.

- L'existence de trois places exactement qui se ramifient dans $k / \mathbb{Q}$.

Pour toute la suite $p_{i}$ désignera un nombre premier congru à 1 modulo 4 , et $q_{i}$ un nombre premier congru à 3 modulo 4 .

Ainsi, pour que $k=\mathbb{Q}(\sqrt{d})$ vérifie les conditions du théorème 1.1 , il faut que $d$ soit de la forme :

$$
p_{1} q_{2} q_{3}, p_{1} q_{2}, 2 p_{1} q_{2}, \text { ou } 2 q_{2} q_{3} .
$$

Une conséquence de ceci est que $\operatorname{Gal}(M / \mathbb{Q})=(2,2,2)$.

On a donc la proposition suivante :

Proposition 2.1. Soit $k / \mathbb{Q}$ une extension quadratique réelle dont la norme de l'unité fondamentale est 1 . Supposons que $l_{k}^{+}(2)=(2,2)$. Alors l'extension $k_{1}^{+}(2) / \mathbb{Q}$ est $(2,2,2)$. Par conséquent, les trois sur-extensions quadratiques de $k$ contenues dans $k_{1}^{+}(2)$ sont des extensions bi-quadratiques de $\mathbb{Q}$. Deux de ces corps bi-quadratiques sont imaginaires, le troisième est réel.

Remarque 2.2. On peut noter que pour $k=\mathbb{Q}(\sqrt{d})$, avec $d=p_{1} p_{2} p_{3}$, ou bien $d=2 p_{1} p_{2}$, on a $d_{2} c l_{k}=2$, et dans ce cas, soit la norme de l'unité fondamentale est -1 , soit $d_{4} c l_{k}^{+} \geq 1$.

2.3. Quelques conditions afin que $c l_{k}^{+}(2)=(2,2)$. On est amené à montrer que le rang d'une certaine matrice (vue multiplicativement) est 2 (cf. $[1])$. 
Pour $a$ un nombre premier, $(., .)_{a}$ désignera le symbole de Hilbert, et $(\dot{ })$ le symbole de Legendre.

2.3.1. Cas où $d=p_{1} q_{2} q_{3}$. On a $c l_{k}^{+}(2)=(2,2)$ si et seulement si le rang de la matrice suivante est égal à 2 (notation multiplicative) :

$$
\left(\begin{array}{ccc}
\left(p_{1}, d\right)_{p_{1}} & \left(q_{2}, d\right)_{p_{1}} & \left(q_{3}, d\right)_{p_{1}} \\
\left(p_{1}, d\right)_{q_{2}} & \left(q_{2}, d\right)_{q_{2}} & \left(q_{3}, d\right)_{q_{2}} \\
\left(p_{1}, d\right)_{q_{3}} & \left(q_{2}, d\right)_{q_{3}} & \left(q_{3}, d\right)_{q_{3}}
\end{array}\right) \sim\left(\begin{array}{ccc}
\left(\frac{q_{3}}{p_{1}}\right) & \left(\frac{q_{2}}{p_{1}}\right) & 1 \\
-\left(\frac{q_{3}}{q_{2}}\right) & -\left(\frac{q_{2}}{p_{1}}\right)\left(\frac{q_{3}}{p_{2}}\right) & -1 \\
1 & 1 & 1
\end{array}\right)
$$

Ainsi, $c l_{k}^{+}(2)=(2,2)$ si et seulement si $\left(\frac{q_{2}}{p_{1}}\right)=-1$ ou $\left(\frac{q_{3}}{p_{1}}\right)=-1$.

2.4. Cas où $d=p_{1} q_{2}$. On obtient la matrice suivante :

$$
\left(\begin{array}{ccc}
\left(\frac{2}{p_{1}}\right) & \left(\frac{q_{2}}{p_{1}}\right) & 1 \\
\left.\frac{2}{q_{2}}\right) & -\left(\frac{q_{2}}{p_{1}}\right) & -1 \\
1 & 1 & 1
\end{array}\right)
$$

Ainsi, $c l_{k}^{+}(2)=(2,2)$ si et seulement si $\left(\frac{q_{2}}{p_{1}}\right)=-1$ ou $\left(\frac{2}{p_{1}}\right)=-1$.

2.5. Cas où $d=2 q_{1} a, a$ premier congru à 1 ou 3 modulo 4. On obtient la matrice :

$$
\left(\begin{array}{ccc}
\left(\frac{2}{a}\right) & \left(\frac{q_{1}}{a}\right) & \left(\frac{-1}{a}\right) \\
\left(\frac{2}{q_{1}}\right) & -\left(\frac{2 * a}{q_{1}}\right) & -1 \\
1 & 1 & 1
\end{array}\right)
$$

En particulier, pour $a=p_{2}$, si $\left(\frac{q_{1}}{p_{2}}\right)=-1$ ou bien si $\left(\frac{2}{p_{2}}\right)=-1$, alors $c l_{k}^{+}(2)=(2,2)$.

\section{Le NOYAU DE CAPITUlation}

Soit $K / k$ une extension galoisienne non ramifiée aux places finies ; $G=$ $\operatorname{Gal}(K / k)$.

Nous noterons $\gamma$ l'ensemble des places réelles de $k$ qui se complexifient dans $K / k$ (i.e $\gamma$ est l'ensemble des places archimédiennes de $k$ qui ont un groupe de décomposition non trivial dans $K / k$ ), puis $J_{K / k}$ le noyau de capitulation de $c l_{k}^{+}$dans $c l_{K}^{+}$, plus précisément $J_{K / k}=k e r\left(c l_{k}^{+} \rightarrow c l_{K}^{+}\right)$, l'homomorphisme étant l'inclusion.

Soit $E_{k}^{\gamma}$ le sous-groupe de $E_{k}$ constitué des unités de $k$ qui sont positives en dehors de $\gamma$. Considèrons alors l'application $\Phi$ suivante :

$$
\begin{aligned}
\Phi: E_{k}^{\gamma} / E_{k}^{+} & \rightarrow \prod_{i=1}^{|\gamma|} \mathbb{R}^{*} / \mathbb{R}^{*+} \\
x & \mapsto \quad\left(\sigma_{i}(x)\right)_{i}
\end{aligned}
$$


où les $\sigma_{i}$ sont les plongements associés aux places de $k$ qui se complexifient dans $K / k$.

Enfin notons $\Gamma$ la co-image de $\Phi$.

On a la suite exacte (cf. [6]) :

$$
1 \longrightarrow \Gamma \longrightarrow J_{K / k} \longrightarrow H^{1}\left(G, E_{K}^{+}\right) \longrightarrow 1
$$

On en déduit la proposition suivante :

Proposition 3.1. Soit $k$ un corps quadratique réel.

Si $K / k$ est quadratique imaginaire ( $K$ est totalement imaginaire, et $[K$ : $k]=2$ ), et si de plus l'unité fondamentale de $k$ est totalement positive, alors

$$
\left|J_{K / k}\right|=\left[E_{k}: N_{K / k} E_{K}\right] .
$$

Si $K / k$ est quadratique réel ( $K$ est réel, et $[K: k]=2$ ), alors

$$
\left|J_{K / k}\right|=2 \times\left[E_{k}^{+}: N_{K / k} E_{K}^{+}\right] \text {. }
$$

\section{Preuve}

L'extension $K / k$ étant cyclique, on a [4] :

$$
\left|H^{1}\left(G, E_{K}^{+}\right)\right|=\left|H^{0}\left(G, E_{K}^{+}\right)\right| \times \frac{[K: k]}{2^{|\gamma|}} .
$$

Ainsi il vient avec (4) :

$$
\left|J_{K / k}\right|=\frac{\left|H^{0}\left(G, E_{K}^{+}\right)\right| \times[K: k] \times|\Gamma|}{2^{|\gamma|}}
$$

Il suffit ensuite de remarquer les deux points suivants :

- Si $K / k$ est imaginaire, alors $|\gamma|=2,|\Gamma|=\frac{2^{2}}{\left[E_{k}: E_{k}^{+}\right]}=2$ (car l'unité fondamentale de $k$ est totalement positive), et $\left|H^{0}\left(G, E_{K}^{+}\right)\right|=\left[E_{k}: N_{K / k} E_{K}\right]$ ( $K$ étant imaginaire, $E_{K}^{+}=E_{K}$ ).

- Si $K / k$ est réel, alors $\gamma=\emptyset,|\Gamma|=1$, et $\left|H^{0}\left(G, E_{K}^{+}\right)\right|=\left[E_{k}^{+}: N_{K / k} E_{K}^{+}\right]$.

\section{UN RAPPEL DE THÉORIE DES GROUPES}

On peut trouver ce résultat dans [2] ou [5].

Proposition 4.1. Soit $G$ un 2-groupe fini, tel que $G^{a b}=(2,2)$. Alors $G$ est l'un des groupes suivants : le groupe abélien $(2,2)$, le groupe dihedral $D_{n}$, le groupe semi-dihedral $S_{n}$ ou le groupe quaternonien $Q_{n}$.

Notons $H_{i}, i=1,2,3$, les trois sous-groupes distingués de $G$ d'indice 2.

Alors $G=(2,2)$ si et seulement si le noyau de transfert de $G^{a b}$ dans deux des trois groupes $H_{i}^{a b}$ est d'ordre 4. Dans ce cas, l'ordre du noyau de transfert de $G^{a b}$ dans le troisième groupe abélianisé $H_{i}^{a b}$ est nécessairement 4. 


\section{Un RAPPEL D'Un RÉSUltat DE KubOda [3]}

Pour une extension quadratique $N / \mathbb{Q}$, si $\varepsilon$ est une unité de $k$ de norme 1 , on définit l'entier $\delta_{\varepsilon}$ par

1. $\delta_{\varepsilon}=1$, si $\varepsilon=1$.

2. $\delta_{\varepsilon}=-d$, si $\varepsilon=-1$, et $N=\mathbb{Q}(\sqrt{d}), d>0$ ou $d<0$.

3. $\delta_{\varepsilon}=2$, si $\varepsilon=\sqrt{-1}$, et $N=\mathbb{Q}(\sqrt{-1})$.

4. $\delta_{\varepsilon}=3$, si $\varepsilon=-\zeta, \zeta$ étant une racine primitive cubique de $1 ; \delta_{\zeta}=1$. (ici $N=\mathbb{Q}(\sqrt{-3})$ )

5. Si $\varepsilon$ n'est pas une racine de l'unité, $\delta_{\varepsilon}$ est tel que $\varepsilon+1 / \varepsilon+2 \in \delta_{\varepsilon} \mathbb{Q}^{2}$.

Pour $k$ quadratique réel, on définit $\delta_{k}=\delta_{\varepsilon}$, où $\varepsilon$ est l'unité fondamentale de $k$ lorsque celle-ci est de norme 1 . Dans ce cas particulier, on a $\delta_{k} \neq 1, d$. A noter aussi que $\delta_{k}$ divise $\operatorname{Disc}(k)$.

Dans le cas où la norme de l'unité fondamentale de $k$ est -1 , on définit $\delta_{k}$ comme étant égal à $\delta_{\varepsilon^{2}}$.

On notera $S_{k}$ et $T_{k}$ les quantités suivantes :

$$
\begin{aligned}
& S_{k}=\varepsilon+1 / \varepsilon+2, \\
& T_{k}=\varepsilon+1 / \varepsilon-2,
\end{aligned}
$$

lorsque $\varepsilon$ sera l'unité fondamentale de $k$ de norme 1 (sinon, on prend $\varepsilon^{2}$ ).

On peut remarquer que $S_{k} \in \delta_{k} \mathbb{Q}^{2}$.

On a la proposition suivante :

Proposition 5.1. Si $k=\mathbb{Q}(\sqrt{d}), T_{k} \in d \delta_{k} \mathbb{Q}^{2}$.

\section{Preuve}

Comme $\varepsilon$ est de norme 1, par le théorème 90 de Hilbert, il existe $\alpha \in k$, tel que $\varepsilon=\alpha / \alpha^{\tau}$, où $G a l(k / \mathbb{Q})=\langle\tau\rangle$. A noter que $\delta_{\varepsilon}=N_{k / \mathbb{Q}}(\alpha)$.

Ainsi

$$
T_{k}=\left(\alpha-\alpha^{\tau}\right)^{2} /\left(N_{k / \mathbb{Q}}(\alpha)\right) \in d \delta_{k} \mathbb{Q}^{2}
$$

Pour $k$ quadratique imaginaire, on définit $\delta_{k}=\delta_{\varepsilon}$, où $\varepsilon$ est une racine primitive de l'unité contenue dans $k$.

On a la proposition suivante (cf. [3]) :

Proposition 5.2. Soient $L$ une extension bi-quadratique de $\mathbb{Q}, k_{i}$ les trois sous-corps quadratiques, $\varepsilon_{i}$ des unités des corps $k_{i}$ de norme 1 , et $\delta_{i}$ les entiers attachés à ces unités.

Alors $\prod_{i} \varepsilon^{a_{i}} \in L^{2}$ si et seulement si $\prod_{i} \delta_{i}^{a_{i}} \in L^{2}$. 


\section{DÉMONSTRATION DU THÉORÈME}

6.1. Situation. Soit $k=\mathbb{Q}(\sqrt{d})$ un corps quadratique réel satisfaisant $c l_{k}^{+}(2)=(2,2)$, et dont la norme de l'unité fondamentale est 1 . Notons $\varepsilon_{k}$ l'unité fondamentale de $k$.

Soient $F$ le 2-corps de Hilbert de $k$, et $L$ une des deux sur-extension quadratique imaginaire de $k ; k_{1}^{+}=F L$. Nous noterons $L^{*}$ la seconde extension quadratique imaginaire de $k$.

On distingue 2 cas : le cas où $\sqrt{-1}$ est ni dans $L$, ni dans $L^{*}$ (cas I), et le cas où $L$ (ou $\left.L^{*}\right)$ contient $\sqrt{-1}$ (cas II).

6.1.1. Cas $I$. On sait que l'unité fondamentale de $L$ (et de $L^{*}$ ) est $\varepsilon_{k}$, ou $\sqrt{-\varepsilon_{k}}$ (cf. [3]). Si l'unité fondamentale de $L$ est $\varepsilon_{k}$, alors d'après la proposition 3.1, $\left|J_{L / k}\right|=4$. Sinon, $L$ contient $\sqrt{-\varepsilon_{k}}$, et ainsi $L=k\left(\sqrt{-\varepsilon_{k}}\right)$. Par conséquent, l'unité fondamentale de $L^{*}$ est nécessairement $\varepsilon_{k}$, et $\left|J_{L^{*} / k}\right|=4$.

6.1.2. Cas $I I$. Supposons que $L$ contient $\sqrt{-1} ; L=\mathbb{Q}\left(\sqrt{-1}, \sqrt{-p_{1} q_{2}}\right)$.

On sait alors que l'unité fondamentale de $L$ est $\varepsilon_{k}$ ou bien $\sqrt{\sqrt{-1} \varepsilon_{k}}$ (cf. [3]). Si c'est $\varepsilon_{k}$, alors $\left|J_{L / k}\right|=4$.

Montrons que si l'unité fondamentale de $L$ est $\sqrt{\sqrt{-1} \varepsilon_{k}}$, alors l'unité fondamentale de $L^{*}$ est $\varepsilon_{k}$ :

Dans $L$, notons $k_{1}=\mathbb{Q}\left(\sqrt{-p_{1} q_{2}}\right)$, et $k_{2}=\mathbb{Q}(\sqrt{-1})$. On a $\delta_{k_{1}}=p_{1} q_{2}$, et $\delta_{k_{2}}=2$ (cf. $\S 5$ ). Comme $\sqrt{\sqrt{-1} \varepsilon_{k}}$ est l'unité fondamentale de $L$, il vient que $\delta_{k} \delta_{k_{2}}$ est un carré dans $L$, ainsi nécessairement $\delta_{k}=2$ ou $2 p_{1} q_{2}$ (proposition $5.2)$.

Regardons ensuite dans $L^{*}:$ Notons $k_{3}=\mathbb{Q}\left(\sqrt{-p_{1}}\right), k_{4}=\mathbb{Q}\left(\sqrt{-q_{2}}\right)$. Alors $\delta_{k_{3}}=p_{1}$ et $\delta_{k_{4}}=q_{2}$. Ainsi $\delta_{k} \delta_{k_{3}}^{i} \delta_{k_{4}}^{j}$ n'est pas un carré dans $L^{*}$, donc $-\varepsilon_{k}$ n'est pas un carré dans $L^{*}$. Par conséquent $\varepsilon_{k}$ est l'unité fondamentale de $L^{*}$.

\section{ETUde D'UN CAS PARTICULIER}

Reprenons le cas où $k=\mathbb{Q}\left(\sqrt{p_{1} q_{2} q_{3}}\right),\left(\frac{q_{2}}{p_{1}}\right)=-1$.

Dans cette partie on notera $k_{1}=\mathbb{Q}\left(\sqrt{p_{1}}\right), k_{2}=\mathbb{Q}\left(\sqrt{q_{2} q_{3}}\right), \varepsilon_{1}, \varepsilon_{2}, \varepsilon_{k}$ les unités fondamentales de $k_{1}$, de $k_{2}$ et de $k$. $F$ désignera le corps biquadratique $k_{1} k_{2}$, c'est à dire $F$ est le 2-corps de Hilbert de $k$.

On propose de montrer que le théorème 1.1, permet de donner des renseignements sur le signe des plongements de certaines unités de $F$.

On notera $\sigma_{i}$ les quatres plongements de $F$ définis par:

i) $\sigma_{1}$ est le plongement identité de $F$, 
ii) $\sigma_{2}\left(\sqrt{p_{1}}\right)=-\sqrt{p_{1}}$, et $\sigma_{2}\left(\sqrt{q_{2} q_{3}}\right)=\sqrt{q_{2} q_{3}}$,

iii) $\sigma_{3}\left(\sqrt{p_{1}}\right)=-\sqrt{p_{1}}$, et $\sigma_{3}\left(\sqrt{q_{2} q_{3}}\right)=-\sqrt{q_{2} q_{3}}$,

iv) $\sigma_{4}\left(\sqrt{p_{1}}\right)=\sqrt{p_{1}}$, et $\sigma_{4}\left(\sqrt{q_{2} q_{3}}\right)=-\sqrt{q_{2} q_{3}}$.

On posera $L=\mathbb{Q}\left(\sqrt{-q_{2}}, \sqrt{-p_{1} q_{3}}\right)$, et $L^{*}=\mathbb{Q}\left(\sqrt{-q_{3}}, \sqrt{-p_{1} q_{2}}\right)$.

On a le lemme suivant :

Lemme 7.1.

1) $S i\left(\frac{q_{2}}{p_{1}}\right)^{=}=\left(\frac{q_{3}}{p_{1}}\right)=-1$, alors $\sqrt{\varepsilon_{k}} \in F$.

2) $S i\left(\frac{q_{2}}{p_{1}}\right)=-1$ et $\left(\frac{q_{3}}{p_{1}}\right)=1$, alors $\sqrt{-\varepsilon_{k}} \in L^{*}=\mathbb{Q}\left(\sqrt{-q_{3}}, \sqrt{-p_{1} q_{2}}\right)$.

\section{Preuve}

Notons $k_{3}=\mathbb{Q}\left(\sqrt{-q_{2}}\right)$ et $k_{4}=\mathbb{Q}\left(\sqrt{-p_{1} q_{3}}\right)$. Alors $\delta_{k_{3}}=q_{2}$ et $\delta_{k_{4}}=p_{1} q_{3}$.

De $\left(\frac{q_{2}}{p_{1}}\right)=-1$, on a

$$
\left(q_{2}, d\right)_{p_{1}}=\left(p_{1} q_{3}, d\right)_{p_{1}}=\left(p_{1}, d\right)_{q_{2}}=-1
$$

ainsi, $\delta_{k}$ est égal soit à $q_{3}$, soit à $p_{1} q_{2}$, soit à $q_{2} q_{3}$. On peut alors remarquer que $\delta_{k} \delta_{k_{3}}^{i} \delta_{k_{4}}^{j}$ n'est pas un carré dans $L$, ainsi $\sqrt{-\varepsilon_{k}}$ n'est pas dans $L$.

1) Si de plus, $\left(\frac{q_{3}}{p_{1}}\right)=-1$, alors $\delta_{k}=q_{2} q_{3}$, c'est donc un carré dans $F$. Ainsi, $\sqrt{\varepsilon_{k}} \in F$.

2) $\mathrm{Si}\left(\frac{q_{3}}{p_{1}}\right)=1$, alors $\delta_{k}=q_{3}$ ou $p_{1} q_{2}$. On voit alors que $-\varepsilon_{k}$ est un carré dans $L^{*}$, ainsi $\sqrt{-\varepsilon_{k}} \in L^{*}$.

7.1. Supposons : $\left(\frac{q_{2}}{p_{1}}\right)=\left(\frac{q_{3}}{p_{1}}\right)=-1$. D'après le lemme précédent, $\varepsilon$ est l'unité fondamentale de $L$ et de $L^{*}$. Alors $\left|J_{L / k}\right|=\left|J_{L^{*} / k}\right|=4$. Dans ce cas, $\left|J_{F / k}\right|=4$, ceci d'après la proposition 4.1 .

De plus, notons que :

$$
N_{F / k}\left(\varepsilon_{1} \sqrt{\varepsilon_{k}}\right)=N_{k_{1} / \mathbb{Q}}\left(\varepsilon_{1}\right) N_{k / \mathbb{Q}}\left(\sqrt{\varepsilon_{k}}\right)=-\left(-\varepsilon_{k}\right)=\varepsilon_{k},
$$

où $\varepsilon_{1}$ est l'unité fondamentale de $\mathbb{Q}\left(\sqrt{p_{1}}\right)$, de norme égale donc à -1 .

Il vient ainsi le corollaire suivant :

Corollaire 7.2. Supposons $\left(\frac{q_{2}}{p_{1}}\right)=\left(\frac{q_{3}}{p_{1}}\right)=-1$, et notons $\varepsilon_{k}$ et $\varepsilon_{1}$ les unités fondamentales de $k=\mathbb{Q}\left(\sqrt{p_{1} q_{2} q_{3}}\right)$ et de $k_{1}=\mathbb{Q}\left(\sqrt{p_{1}}\right)$. Alors l'unité $\varepsilon_{1} \sqrt{\varepsilon_{k}}$ vue dans le compositum des 2 corps quadratiques, n'est pas totalement positive.

Nous allons donner deux preuves de ce résultat.

Preuve I

Rappelons que si $c l_{k}(2)=(2)$, alors $E_{k}=N_{F / k} E_{F}$ (cf. [5], [6]). En particulier, $\varepsilon_{k}$ est norme d'unité dans l'extension $F / k$.

$\operatorname{De}\left|J_{F / k}\right|=4$, on a $\left[E_{k}^{+}: N_{F / k} E_{F}^{+}\right]=2$ (proposition 3.1). Ainsi il vient que $\varepsilon_{k}$ n'est pas norme dans $F / k$ d'une unité de de $F$ totalement positive. 


\section{Preuve II}

On rappelle que $\delta_{k}=q_{2} q_{3}$. D'après la proposition $5.1, \sqrt{S_{k}}=a \sqrt{q_{2} q_{3}}$ et $\sqrt{T_{k}}=b \sqrt{p_{1}}, a, b \in \mathbb{Q}$.

D'autre part, on connait $\sqrt{\varepsilon_{k}}$ (cf. [3]) :

$$
\sqrt{\varepsilon_{k}}=\frac{\sqrt{S_{k}}+\sqrt{T_{k}}}{2} \text {. }
$$

Ainsi $\sigma_{2}\left(\sqrt{S_{k}}+\sqrt{T_{k}}\right)$ est positif, et $\sigma_{2}\left(\varepsilon_{1}\right)$ est négatif, car $\varepsilon_{1}$ est de norme $-1$.

Donc $\sigma_{2}\left(\varepsilon_{1} \sqrt{\varepsilon_{k}}\right)$ est négatif.

7.2. Supposons : $\left(\frac{q_{2}}{p_{1}}\right)=-1,\left(\frac{q_{3}}{p_{1}}\right)=1$. D'après le lemme $7.1, \sqrt{-\varepsilon_{k}} \in$ $L^{*}$, où $L^{*}=\mathbb{Q}\left(\sqrt{-q_{3}}, \sqrt{-p_{1} q_{2}}\right)$. Ainsi $\left|J_{L^{*} / k}\right|=2$, et $\left|J_{L / k}\right|=4$.

Par la proposition 5.2, on a donc nécessairement $\left|J_{F / k}\right|=2$.

Avant de poursuivre, notons le lemme suivant :

Lemme 7.3. $S i\left(\frac{q_{2}}{q_{3}}\right)=1$, alors $\delta_{k}=p_{1} q_{2}$, et $\delta_{k_{2}}=q_{2}$.

Si $\left(\frac{q_{2}}{q_{3}}\right)=-1$, alors $\delta_{k}=\delta_{k_{2}}=q_{3}$.

Dans ces deux cas, $\sqrt{\varepsilon_{k} \varepsilon_{2}} \in F$.

On peut alors remarquer que

$$
N_{F / k}\left(\sqrt{\varepsilon_{k} \varepsilon_{2}}\right)^{2}=N_{k_{2} / \mathbb{Q}}\left(\varepsilon_{2}\right) N_{F / k}\left(\varepsilon_{k}\right)=\varepsilon_{k}^{2} .
$$

Ainsi,

$$
\varepsilon_{k}=N_{F / k}\left(\sqrt{\varepsilon_{k} \varepsilon_{2}}\right)
$$

ou bien

$$
\varepsilon_{k}=N_{F / k}\left(\varepsilon_{1} \sqrt{\varepsilon_{k} \varepsilon_{2}}\right) .
$$

On a alors le corollaire suivant :

Corollaire 7.4. Si $\left(\frac{q_{2}}{q_{3}}\right)=1$, alors $\varepsilon_{k}=N_{F / k}\left(\varepsilon_{1} \sqrt{\varepsilon_{k} \varepsilon_{2}}\right)$, et $\varepsilon_{1} \sqrt{\varepsilon_{k} \varepsilon_{2}}$ est totalement positive.

$S i\left(\frac{q_{2}}{q_{3}}\right)=-1$, alors $\varepsilon_{k}=N_{F / k}\left(\sqrt{\varepsilon_{k} \varepsilon_{k_{2}}}\right)$, et $\sqrt{\varepsilon_{k} \varepsilon_{k_{2}}}$ est totalement positive.

\section{Preuve}

Nous allons montrer uniquement le premier point.

Supposons que $\left(\frac{q_{2}}{q_{3}}\right)=1$, alors d'après le lemme 7.4 et la proposition 5.1, on a

$$
\begin{aligned}
\sqrt{\varepsilon_{k}} & =1 / 2\left(\sqrt{S_{k}}+\sqrt{T_{k}}\right) \\
& =a \sqrt{p_{1} q_{2}}+b \sqrt{q_{3}}
\end{aligned}
$$

et

$$
\begin{aligned}
\sqrt{\varepsilon_{2}} & =1 / 2\left(\sqrt{S_{k_{2}}}+\sqrt{T_{k_{2}}}\right) \\
& =a^{\prime} \sqrt{q_{2}}+b^{\prime} \sqrt{q_{3}}
\end{aligned}
$$


où $a, b, a^{\prime}, b^{\prime}$ sont dans $\mathbb{Q}$.

Il vient alors,

Ainsi

$$
\begin{aligned}
\sqrt{\varepsilon_{2} \varepsilon_{k}} & =1 / 4\left(\sqrt{S_{k}} \sqrt{S_{k_{2}}}+\sqrt{S_{k}} \sqrt{T_{k_{2}}}+\sqrt{T_{k}} \sqrt{S_{k_{2}}}+\sqrt{T_{k}} \sqrt{T_{k_{2}}}\right) \\
& =a a^{\prime} q_{2} \sqrt{p_{1}}+a b^{\prime} \sqrt{p_{1} q_{2} q_{3}}+b a^{\prime} \sqrt{q_{2} q_{3}}+b b^{\prime} q_{3} .
\end{aligned}
$$

$\sigma_{2}\left(\sqrt{\varepsilon_{2} \varepsilon_{k}}\right)=1 / 4\left(\sqrt{S_{k_{2}}}\left(\sqrt{T_{k}}-\sqrt{S_{k}}\right)+\sqrt{T_{k_{2}}}\left(\sqrt{T_{k}}-\sqrt{S_{k}}\right)\right)$

$\sigma_{3}\left(\sqrt{\varepsilon_{2} \varepsilon_{k}}\right)=1 / 4\left(\sqrt{S_{k}}\left(\sqrt{T_{k_{2}}}-\sqrt{S_{k_{2}}}\right)+\sqrt{T_{k}}\left(\sqrt{T_{k_{2}}}-\sqrt{S_{k_{2}}}\right)\right)$.

$\sigma_{4}\left(\sqrt{\varepsilon_{2} \varepsilon_{k}}\right)=1 / 4\left(\left(\sqrt{S_{k}}-\sqrt{T_{k}}\right)\left(\sqrt{S_{k_{2}}}-\sqrt{T_{k_{2}}}\right)\right)$.

On peut alors noter que $\sigma_{2}\left(\sqrt{\varepsilon_{2} \varepsilon_{k}}\right)$ et $\sigma_{3}\left(\sqrt{\varepsilon_{2} \varepsilon_{k}}\right)$ sont négatifs, ainsi $\varepsilon_{k}=$ $\varepsilon_{1} \sqrt{\varepsilon_{k} \varepsilon_{2}}$. Il apparait alors que les quatre plongements de $\varepsilon_{1} \sqrt{\varepsilon_{2} \varepsilon_{k}}$ sont positifs.

Remarque 7.5. On peut également montrer le corollaire 7.3 sur le modèle de la preuve I du corollaire 7.2.

En effet, puisque $\left|J_{L / k}\right|=2$, d'après la proposition 3.1, $\left[E_{k}^{+}: N_{F / k} E_{F}^{+}\right]=1$. Ainsi $\varepsilon_{k}$ est norme d'unité positive. Il suffit ensuite de remarquer que $\varepsilon_{k}$ ne peut être norme, dans $F / k$, que de $\varepsilon_{1} \sqrt{\varepsilon_{k} \varepsilon_{2}}$ ou de $\sqrt{\varepsilon_{k} \varepsilon_{2}}$.

7.3. Illustrations. Nous allons donner deux exemples qui illustrent le corollaire 7.3.

7.3.1. Premier exemple. Prenons $k=\mathbb{Q}(\sqrt{5 * 19 * 23}), k_{1}=\mathbb{Q}(\sqrt{5}), k_{2}=$ $\mathbb{Q}(\sqrt{19 * 23})\left(p_{1}=5, q_{2}=23\right.$, et $\left.q_{3}=19\right)$.

L'unité fondamentale de $k$ est

$$
\varepsilon_{k}=2447977239109027+107029473520104(1+\sqrt{5 * 19 * 23}) / 2 .
$$

On a

$$
\sqrt{\varepsilon_{k}}=3297886 \sqrt{5 * 23}+8113491 \sqrt{19}
$$

L'unité fondamentale de $k_{2}$ est

$$
\varepsilon_{2}=10+(1+\sqrt{19 * 23}) / 2 \text {, }
$$

et

$$
\sqrt{\varepsilon_{2}}=\frac{1}{2}(\sqrt{19}+\sqrt{23})
$$

On a donc

$\sqrt{\varepsilon_{k} \varepsilon_{2}}=\frac{1}{2}(154156329+75851378 \sqrt{5}+8113491 \sqrt{19 * 23}+3297886 \sqrt{5 * 19 * 23})$.

On note alors que

$$
N_{F / k}\left(\varepsilon_{1} \sqrt{\varepsilon_{k} \varepsilon_{2}}\right)=\varepsilon_{k} .
$$

Ainsi, d'après le corollaire 7.3, $\varepsilon_{1} \sqrt{\varepsilon_{k} \varepsilon_{2}}$ est totalement positive.

Voici les quatres plongements de cette unité :

i) $\sigma_{1}\left(\varepsilon_{1} \sqrt{\varepsilon_{k} \varepsilon_{2}}\right) \approx 847628209.995$

ii) $\sigma_{2}\left(\varepsilon_{1} \sqrt{\varepsilon_{k} \varepsilon_{2}}\right) \approx 0.0000000647$

iii) $\sigma_{3}\left(\varepsilon_{1} \sqrt{\varepsilon_{k} \varepsilon_{2}}\right) \approx 15452508.395$

iv) $\sigma_{4}\left(\varepsilon_{1} \sqrt{\varepsilon_{k} \varepsilon_{2}}\right) \approx 0.00000000808$ 
7.3.2. Second exemple. On prend $\mathbb{Q}(\sqrt{5 * 7 * 11})$.

L'unité fondamentale de $k$ est

$$
\varepsilon_{k}=90947+9768 *(1+\sqrt{5 * 7 * 11}) / 2 .
$$

On a

$$
\sqrt{\varepsilon_{k}}=37 \sqrt{5 * 7}+66 \sqrt{11} .
$$

L'unité fondamentale de $k_{2}=\mathbb{Q}(\sqrt{7 * 11})$ est

$$
\varepsilon_{2}=4+(1+\sqrt{7 * 11}) / 2 \text {, }
$$

et

$$
\sqrt{\varepsilon_{2}}=\frac{1}{2}(\sqrt{7}+\sqrt{11})
$$

On a ainsi

$$
\sqrt{\varepsilon_{k} \varepsilon_{2}}=\frac{1}{2}(726+259 \sqrt{5}+66 \sqrt{7 * 11}+37 \sqrt{5 * 7 * 11}) .
$$

On note alors que

$$
N_{F / k}\left(\sqrt{\varepsilon_{k} \varepsilon_{2}}\right)=\varepsilon_{k}
$$

L'unité $\sqrt{\varepsilon \varepsilon_{2}}$ est donc totalement positive. En voici les quatres plongements

i) $\sigma_{1}\left(\sqrt{\varepsilon_{k} \varepsilon_{2}}\right) \approx 1305.140$

ii) $\sigma_{2}\left(\sqrt{\varepsilon_{k} \varepsilon_{2}}\right) \approx 0.00680$

iii) $\sigma_{3}\left(\sqrt{\varepsilon_{k} \varepsilon_{2}}\right) \approx 146.851$

iv) $\sigma_{4}\left(\sqrt{\varepsilon_{k} \varepsilon_{2}}\right) \approx 0.000766$

\section{REFERENCES}

[1] G. Gras, Sur les l-Classes d'idéaux dans les extensions cycliques relatives de degré premier l, Ann. Inst. Fourier 23, fasc. 3 et 4 (1973).

[2] H. Kisilevsky, Number fields with Class Number congruent to 4 mod 8 and Hilbert's Theorem 94, J. Number Theory 8 (1976), 271-279.

[3] T. Kubota, Über den Bizyklischen Biquadratischen Zahlkörper, Nagoya Math. J. 10 (1955), 65-85.

[4] S. Lang, Algebraic Number Theory, Addison Wesley, New York, 1970.

[5] F. Lemmermeyer, Construction of Hilbert Class Field II, preprint.

[6] C. Maire, T-S capitulation, Publ. Math. fac. Sci. de Besançon (94-95).

Ch. Maire

Laboratoire A2X - Université Bordeaux I

351, Cours de la Libération - 33405 Talence Cedex - France

Email : maire@math.u-bordeaux.fr 